Die Zahlen weichen alle $a b$ in der $z \mathfrak{u}$ erwartenden Richtung. Erhöhte Temperatur, grösserer Ueberschuss, geringere Verdiinnung geben grössere Jodzahlen. Man hat es hier deutlich mit dem auch in den Tabeilen I, III, V, VII zu erblickenden Rückgang nach Erreichung der Minimumgeschwindigkeit oder einer Geschwindigkeit, die practisch gleich Null $z u$ setzen ist, $z \mathfrak{u}$ thun. Wie schon gesagt, muss dieser vielleicht für einen immer kleiner werdenden 'Theil noch der Addition zugeschrieben werden, zu einem anderen Theile hat er aber sicher eine andere Ursache, z. B. eine directe Oxydation des Fetts oder dessen Unreinlichkeiten durch die unterjodige Säure.

Um zu entscheiden, in wie weit die erzielten Jodzahlen wirklich das Maas bilden für die in den Fetten vorkommenden Doppelbindungen, habe ich mittelst Jodchlorid-Eisessiglösung die Jodzahlen einiger ungesättigten Säuren bestimmt. Das Resultat giebt folgende

'Tabelle XII

\begin{tabular}{|c|c|c|c|c|c|}
\hline & \multirow{2}{*}{$\mid \begin{array}{c}\text { Schmelz- } \\
\text { punkt }\end{array}$} & \multicolumn{2}{|c|}{ Säurezahl } & \multicolumn{2}{|c|}{ Jodzahl } \\
\hline & & $\begin{array}{c}\text { be- } \\
\text { rechnet }\end{array}$ & $\begin{array}{c}\text { ge- } \\
\text { funden }\end{array}$ & $\begin{array}{c}\text { be- } \\
\text { rechnet }\end{array}$ & $\begin{array}{c}\text { ge- } \\
\text { funden }\end{array}$ \\
\hline Erucasäure. & 32 & 165,7 & 165,2 & 75,15 & 74,9 \\
\hline Brassidinsàure . & 60 & 165,7 & 165,0 & 75, I 5 & 75,0 \\
\hline Elaidinsäure & 44 & $19^{8,6}$ & 198,4 & 90,07 & 90,0 \\
\hline Oelsäure ${ }^{1}$ ) & - & I 98,6 & I 84,3 & 90,07 & 87,6 \\
\hline Undecylensäure & - & $304,3 \mid$ & 292,9 & $\left|13^{6,6}\right|$ & I 33, I \\
\hline
\end{tabular}

Die Zahlen der Erucasäure, Brassidinsäure und Elaidinsäure stimmen sehr gut. Die Oelsäure giebt eine etwas $\mathrm{zu}$ niedrige $Z$ ahl, ist aber auch nach der Säurezahl nicht als rein zu betrachten, wenn sie auch bedeutend besser als eine aus andrer Bezugsquelle erhaltene Acidum oleïnic. cryst. war, die eine Säurezahl von 188,8 und eine Jodzahl von 102,5 zeigte. Die Undecylēnsäure war ebensowenig ganz rein. Handels-Undecylensäure aus verschiedenen Bezugsquellen zeigten Jodzahlen von I 20 bis 126 und Säurezahlen von $25 \mathrm{I}-256$, waren also sehr unrein. Durch zweimalige fractionirte Fällung des Bariumsalzes aus Alkohol gelang es mir eine Undecylensäure $z u$ erhalten, die die oben angefïhrten Zahlen zeigte. Die Verunreinigung der Handelspräparate ist aller Wahrscheinlichkeit nach ein Polymerisationsproduct. Eine kurze Erhitzung setzt Jodzahl und Säurezahl dieser Säure erheblich herab; bekanntlich tritt hierbei Polymerisation ein. Wahrscheinlich ist also die untersuchte Undecylen. säure nicht frei vom Polymerisationsproducte gewesen.

Man darf meiner Meinung nach aus diesen Zahlen schliessen, dass bei den Fettsäuren mit nur einer Doppelbindung und bei den Fetten, die grösstentheils nur solche ungesättigte Fettsäuren enthalten, die richtig bestimmte Jodzahl mit der theoretischen genügend übereinstimmt. Ob dies auch für die Linolund Linolensäureu Geltung hat, muss vorläufig dahin. gestellt bleiben.

Delft, Laboratorium der Oliefabriek.

1) Als frei von Linolsäure verkauft.

\title{
Beiträge
}

\section{zur Einführung einer einheitlichen Bestimmung des Talgtiters.}

Von Dr. A. A. Shukoff.

1. Vergleich verschiedener älterer Methoden.

Die Titrirung des Talges wird im Handel hauptsächlich nach den 3 folgenden Methoden ausgeführt:

1. nach Dalican ${ }^{\mathbf{1}}$ ) (Frankreich und Amerika),

2. nach Norman 'Tate') (England, Australien, Amerika),

3. nach Wolfbauer ${ }^{3}$ ) (Oesterreich).

1) Benedikt-Ulzer, Analyse d. Fette, pag. 99; ausfübrlicher Jean, Chimie analytique des matières grasses, pag. 332 .

2) The Examination of Tallow (Privatausgabe, im Handel nicht erschienen) und aus Privatmittheilungen.

3) Benedikt, loc, cit, pag. IOI; ausfủhrlicher: Mittheil. des K. k. Technol. Gewerbemuseums i 894, No. 1-4.
Die Resultate, welche nach diesen verschiedenen Methoden erhalten werden, sind nun leider verschiedene, was ausschliesslich auf die verschiedene Art und Weise bei der Bestimmung der Erstarrungstemperatur der Fettsäuren zurückgeführt werden muss, wie aus dem Folgenden ersichtlich sein wird. Was die Verseifung des Talgs speciell anbetrifft, so wird sie durch wässrige Kalilauge (Wolfbauer), oder durch ein Gemisch von Alkohol und starker Natronlauge (Dalican), oder endlich durch alkoholische Lauge (N. Tate) herbeigeführt.

Wir haben eine grössere Anzahl verschiedener Talgproben nach diesen Methoden verseift und sind zu der Ueberzengung gekommen, dass beim sorg. fältigen Arbeiten die Verseifung nach allen drei 
Arbeitsweisen eine vollkommene ist; in unserem Laboratorium gebrauchen wir ausschliesslich die Dalican'sche Methode wegen ihrer Schnelligkeit und Einfachheit. Wir können bezeugen, dass selbst bei den höchstschmelzenden Hammeltalgen die Verseifung eine leichte und vollkommene ist. Bei den hochschmelzenden Talgen ist es vielleicht am Platze, etwas mehr (10-20 ccm) Alkohol als in der Vorschrift zu gebrauchen, die Verseifung geht dann leichter und sicherer.

Die beim Verseifen des Talgs erhaltenen Fettsäuren müssen unbedingt getrocknet werden. Dits Trocknen der Fettsäuren ist ganz unentbehrlich und wird auch von $N$. Tate und Wolfbauer vorgeschrieben, nicht aber von Dalican. Der Gebrauch von ungetrockneten Fettsäuren giebt nicht nur zu niedrige Werthe, sondern die Angaben werden un. sicher und schwankend, je nach dem Wassergehalt der Probe.

Die hohe Wichtigkeit des Trocknens der Fettsäuren, auf welche schon Wolfbauer aufmerksam gemacht hat, ist aus der folgenden Tabelle leicht zu ersehen.

\begin{tabular}{c|c|c}
\hline \multirow{2}{*}{ Titer nach } & \multicolumn{2}{|c}{ Fettsäuren } \\
& getrocknet & ungetrocknet \\
\hline Wolfbauer & 44,0 & 43,5 \\
$"$ & 43,7 & 43,7 \\
$"$ & $4 \mathrm{I}, 6$ & $4 \mathrm{I}, 5$ \\
Dalican & $4 \mathrm{I}, 5$ & $4 \mathrm{I}, \mathrm{I}$ \\
43,3 & 42,9
\end{tabular}

Die Bestimmung des Talgtiters selbst, d. h. der höchsten bei der Erstarrung beobachteten Temperatur, geschieht bei allen 3 Methoden nach dem. selben Princip: die erstarrenden Fettsäuren werden während des Erstarrens mit dem Thermometer durchgeriuhrt; verschieden ist lediglich die Menge der verwendeten Fettsäuren, die Grösse des Gefässes, sowie die Art und Weise des Durchmischens während des Erstarrens.

Der Einfluss der Weite des Gefässes ist aus der folgenden Tabelle klar zu ersehen:

\begin{tabular}{|c|c|c|c|c|}
\hline \multirow{2}{*}{\multicolumn{2}{|c|}{$\frac{\text { Weite des Gefässes }}{31 / 1 \mathrm{~cm}}$}} & \multicolumn{3}{|c|}{ Titer } \\
\hline & & 49,5 & 45,2 & $4 I, 8$ \\
\hline $23 / 4$ & $"$ & 49,4 & - & - \\
\hline $21 / 9$ & $"$ & 49,3 & - & - \\
\hline 2 & $"$ & 49,2 & - & - \\
\hline I $: 1 / 4$ & $"$ & 49,0 & 44,8 & $4 \times, 5$ \\
\hline I $1 / 2$ & $"$ & 48,9 & - & - \\
\hline
\end{tabular}

Die kleinste Quantität - etwa $30 \mathrm{gr}$ - und das engste Gefäss - I1/2 - $2 \mathrm{~cm}$ - wählt Dalican; etwas mehr N. Tate - $4^{\circ}$ gr. Die Weite seines Gefässes ist untekannt; in unseren Versuchen betrug sie $3 \mathrm{~cm}$. Am meisten Substanz verwendet Wolfbauer - I20 gr - bei einem Gefäss von $3^{1 / 2} \mathrm{~cm}$ Weite. Wie aus folgender Tabelle ersichtlich ist, liefert dementsprechend die höchsten Werthe die Methode von Wolfbauer, die niedrigsten die von Dalican.

\begin{tabular}{cc|c|c|c}
\hline & & \multicolumn{3}{|c}{ Talgtiter nach: } \\
& & Dalican & N. Tate & Wolf baucr \\
\hline Stearin . . . . & $5 \mathrm{I}, 2$ & - & $5 \mathrm{I}, 5$ \\
Hammeltalg . . . & 45,0 & 45,3 & 45,5 \\
Amerik. Knochenfett & $43,4-5$ & 44,0 & $44, \mathrm{I}$ \\
" & & $43, \mathrm{I}-2$ & 43,6 & 43,8 \\
Russisches & " & $4 \mathrm{I}, 5$ & $4 \mathrm{I}, 9$ & 42,0 \\
" & $4 \mathrm{I}, \mathrm{I}-2$ & $4 \mathrm{I}, 5$ & $4 \mathrm{I}, 7$ \\
& & $40,8-9$ & $4 \mathrm{I}, 4$ & $4 \mathrm{I}, 6$
\end{tabular}

Die Daten nach Dalican sind mit getrockneten Fettsäuren erhalten; sie schwanken um $0, I^{0}$, je nachdem man die Weite des Gefässes zu $\mathrm{I} 1 / \mathrm{g}$ oder $2 \mathrm{~cm}$ wählt. Im Allgemeinen muss man noch bemeiken, dass ausser den genannten Factoren (Feuchtigkeitsgrad der Fettsäuren, Weite des Gefässes) noch eine grosse Rolle die Art und Weise des Durchmischens der Fettsäuren während des Erstarrens spielt. Um stimmende Resultate zu erhalten, muss man sich hierbei genau an die eingehenden Angaben der Autoren halten, und selbst dann hatten wir Gelegenheit, uns zu tiberzeugen, dass eine constante persönliche Differen $z$ zwischen den Angaben verschiedener Beobachter vorliegen kann.

Um diesen persönlichen Einfluss auf ein Minimum zu reduciren, und um eine in der Praxis sehr unangenehme und lästige Unbequemlichkeit der sonst vorzüglichen Methode von Wolfbauer zu umgehen, nämlich die etwas grosse Quantität der zu verwendenden Fettsäuren, habe ich gelegentlich des III. Internationalen Congresses fiir angewandte Chemie in Wien eine neue in unserem Laboratorium ausgearbeitete Methode vorgeschlagen, die im Folgenden ausfuhrlich beschrieben werden soll.

2. Eine neue Methode zur Bestimmung der Erstarrungstemperatur der Fettsäuren.

Die Bestimmung der Erstarrungstemperatur von Gemengen verschiedener Körper wird in der physikalischen Chemie sehr oft angewandt, und -die Methoden zu ihrer Bestimmung gehören zu den 
gebräuchlichsten und best ausgearbeiteten - ich meine die Bestimmung der Gefrierpunktserniedrigung verdünnter Lösungen. Es ist wirklich bemerkenswerth, dass diese Methoden bisher in der Fettchemie gar keine Beachtung gefunden haben. Ich habe mich bemüht, die einfachste dieser Methoden, diejenige von Eykman, bei der Bestimmung des Titers anzuwenden.

Nach einigen Versuchen kam ich zur folgenden Apparatenform.

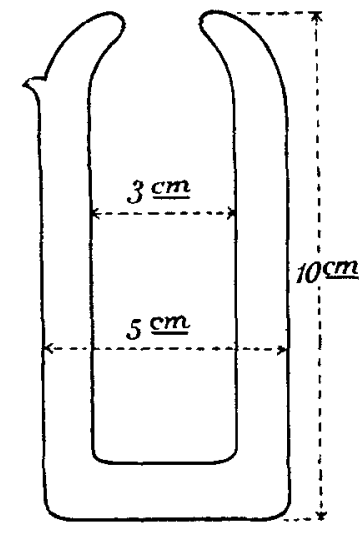

$1 / 2$ natïrl. Grösse

Das Gefäss, welches zur Aufnahme der Fettsäure dient, ist vom sogenannten Dewar'schen Vacuummantel umgeben, $d$. $h$. in ein anderes grösseres Gefäss eingeschmolzen, und zwischen beiden Gefässen eine Crooke'sche Leere hergestellt. Die Evacuirung zwischen den Gefässen ist nicht unumgänglich nothwendig, aber das Arbeiten mit solchem Vacuummantel ist viel angenehmer, da man vor Allem das Gefäss nicht mit Tïchern u. dgl. zu umbiullen braucht, um ein $z u$ rasches Erkalten der Fettsäuren an den Wänden zu verhindern. Die Dimensionen des Apparates sind aus der Zeichnung ersichtlich. ${ }^{1}$ ) Bei der Ausarbeitung des Apparates galt es vor allen Dingen, gemäss der Definition der Erstarrungstemperatur als der höchsten beim Erstarren beobachteten, diejenigen Dimensionen für den Apparat zu wählen, bei welchen wirklich dieser Maximalwerth erreicht wird, und über welche hinaus kein weiteres Steigen der Erstarrungstemperatur herbeigeführt werden kann. Nach eingehenden Controllversuchen wurde gefunden, dass die oben angeführten Dimensionen diesen Bedingungen vollkommen genügen.

Die Bestimmung der Erstarrungstemperatur wird folgendermassen vorgenommen:

In das innere Gefäss werden $30-40 \mathrm{gr}$ (fast

1) Die Ausfihrung des Apparates hat die Firma Max Kähler \& Martini in Berlin übernommen. bis zum Rande) geschmolzene Fettsäuren eingegossen und mittels eines Korkstopfens ein in $1 / \mathrm{s}^{\circ}$ getheiltes Thermometer ${ }^{1}$ ) genau in der Mitte des Gefässes befestigt. Ist die Temperatur auf etwa $5^{\circ}$ oberhalb des erwarteten Erstarrungspunktes gesunken, so fängt man an den Apparat stark und regelmässig von oben nach unten zu schütteln und hört mit dem Schütteln erst dann auf, wenn der Inhalt deutlich triib und undurchsichtig geworden ist; ein etwas spätes Aufhören des Schiittelns hat indessen wenig Bedeutung; übrigens gewöhnt man sich auch rasch, den richtigen Moment zu wählen. Ist man in Bezug auf die Höhe des erwarteten Erstarrungspunkts in Unsicherheit, so kann man einen Vorversuch ausführen, indem man mit dem Schütteln bis zum Erscheinen der ersten Krystalle wartet; man wiederholt dann den Versuch, wie oben beschrieben. Die Werthe, welche man nach dieser Methode erbält, decken sich vollkommen mit denjenigen von Wolfbauer, wie beispielsweise folgende vergleichende Daten zeigen:

\begin{tabular}{c|c}
\hline \multicolumn{2}{|c}{ Talgtiter nach } \\
Wolf bauer & Shukof \\
\hline $5 \mathrm{I}, 4$ & $5 \mathrm{I}, 5$ \\
45,5 & 45,5 \\
44,0 & 44,0 \\
43,8 & 43,8 \\
$4 \mathrm{I}, 7$ & $4 \mathrm{I}, 7$ \\
$4 \mathrm{I}, 6$ & $4 \mathrm{I}, 6$
\end{tabular}

Der Vortheil der Methode vor derjenigen von Wolfbauer liegt $r$ ) in dem vollkommenen Eliminiren vom persönlichen Fehler, und 2) in der kleineren Menge der erforderlichen Fettsäuren. Es ist dies besonders für den Fabrikschemiker von Werth, der nur zu oft in die Lage kommt, kleinere Talgproben, besonders Postproben, auf ihren Titer zu untersuchen.

Die beschriebene Methode lässt sich auch mit Vortheil bei den verschiedensten anderen Körperklassen anwenden. In der folgenden Tabelle sind einige Bestimmungen zusammengestellt, welche in der letzten Zeit in unserem Laboratorium ausgefuhrt wurden. Die Zahlen der ersten Spalte sind die des Erstarrungspunkts, die der zweiten die Erhöhung der Temperatur beim Erstarren.

Von den einzelnen Bestimmungen ist besonders auf diejenigen beim Paraffin aufmerksam zu machen; sie liefern ausserordentlich scharfe und exacte Re-

1) In unserem Laboratorium werden Thermometer nach Art der Baudin'schen gebraucht; die Theilung sind von $0-70^{\circ}$; Gesamimtlänge $20 \mathrm{~cm}$. 
sultate, während die meisten, der jetzt gebrauchten sehr oft höchst willkürlichen Methoden sowohl Mangel an Scharfheit, wie an Gleichförmigkeit der Resultate beklagen lassen.

Die Bestimmungen bei niedrig schmelzenden Körpern (Cocosöl u. a.) dauern viel länger, als diejenigen anderer Substanzen, liefern aber beim sorgfältigen Arbeiten ebenfalls zuverlässige Resultate.

Bei verschiedenen chemischen Präparaten könnte man aus den Abweichungen vom normalen Schmelzpunkt (bei chemischen Individuen sind Schmelzund Erstarrungstemperatur identisch) auf ihren Reinheitsgrad schliessen. Die Schmelzpunkte der käuflichen Diphenylamins and Phenols sind etwas niedriger als die in der Literatur für die reinen Körper niedergelegten.

\begin{tabular}{c|c|c}
\hline \hline & $\begin{array}{c}\text { Erstarrungs- } \\
\text { punkt } \\
0 \mathrm{C} .\end{array}$ & $\begin{array}{c}\text { Temperatur- } \\
\text { zunahme } \\
0 \mathrm{C} .\end{array}$ \\
\hline Hammeltalg, Titer 48,5 & 43,2 & 2,8 \\
$"$ " " & 43,2 & 4,2 \\
Rindertalg, Titer 46,5 & & \\
(für Schmierzwecke) .. & 42,8 & 4,4 \\
Knochenfett, Titer 42,I & $3^{2,6}$ & 0,8 \\
" " " & $3^{2,6}$ & - \\
White Grease, american. & 25,0 & 4,9
\end{tabular}

\begin{tabular}{|c|c|c|}
\hline & $\begin{array}{c}\text { Erstarrungs- } \\
\text { punkt } \\
{ }^{\circ} \mathrm{C} . \\
\end{array}$ & $\begin{array}{c}\text { Temperatur- } \\
\text { zunahme } \\
{ }^{\circ} \mathrm{C} . \\
\end{array}$ \\
\hline $\begin{array}{l}\text { Paraffin schottisch, I I } 8 \\
\text { bis I } 20 \mathrm{~F} . \ldots . .\end{array}$ & 50,4 & $\circ$ \\
\hline Dasselbe . . . . . & $5 \circ, 4$ & $\circ$ \\
\hline Dasselbe ....... & 50,4 & $\circ$ \\
\hline $\begin{array}{l}\text { Paraffin schottisch, I Io } \\
\text { bis I I } 2 \text { F. . . . . . }\end{array}$ & 47,0 & $\circ$ \\
\hline Schuppenparaffin, Galizien & 47,0 & $\circ$ \\
\hline Ceresin . . . . . & 68,0 & 0,3 \\
\hline$" \quad \cdots \cdots \cdot \ldots$ & 67,9 & 0,3 \\
\hline Butterfett. . . . . & 28,6 & $\mathbf{r}, 3$ \\
\hline Cocosöl $\cdot \ldots \cdot \cdots$ & 22,6 & 3,0 \\
\hline$\cdots \cdots$ & 22,7 & - \\
\hline $\begin{array}{l}\text { Chinesisches Pflanzen- } \\
\text { wachs . . . . . . }\end{array}$ & 30,8 & 2,3 \\
\hline Diphenylamin . . . . & 52,7 & $\circ$ \\
\hline$" \quad \cdots \cdot \cdot$ & 52,7 & $\circ$ \\
\hline Phenol ..... & $40, \mathrm{r}$ & $4, \mathrm{I}$ \\
\hline
\end{tabular}

St. Petersburg, December I 898 .

Chemisches Laboratorium der Fabriken

A. M. Shuk off.

\title{
Petroleum als Mittel gegen Pflanzenparasiten.
}

\author{
Von Leopold Gans, Triest.
}

In den letzten Jahren erschienen in den Fachzeitschriften wiederholt Aufsätze, welche die Verwendung des Petroleums als Mittel gegen Pflanzenparasiten behandelten, und es wurden günstige Erfolge constatirt, welche sich bei Benutzung von sogenannten Petroleumbrïhen gegen Insecten mit saugenden Mundwerkzeugen und gegen weichhäutige Schädlinge ergaben.

In dem unlängst erschienenen Handbuch der chemischen Mittel gegenPflanzenkrankheiten von Dr. M. Hollrung wird die Verwendung der Petroleumbrïhen ausfuhrlich besprochen.

Eine Notiz über die günstige Einwirkung von Petroleum zur Bekämpfung der Blattläuse bei den berühmten Rosenstöcken des Hildesheimer Annenfriedhofes brachte Emil K. Blüml. ${ }^{1}$ ) Die Verwendung des Petroleums als Mittel gegen Pflanzenparasiten datirt aber schon seit sehr langer Zeit. Bemerkenswerthe Angaben hieriber giebt auch

(' Chem. Techn. Ztg. 1898, No. 21 .
Heinrich Semmler in seinem Werke: Die tropische Agricultur, I 886, und bespricht da insbesondere die Benutzung der sogen. „Petroleumbutter", einer Emulsion von Petroleum mit saurer Milch. Als grosser Vorzug dieses Mittels wird gerühmt, dass es auch die Insecteneier vernichte.

Die bisher gebräuchlichen Petroleumbrühen werden aus Emulsionen von Petroleum mit Seife und Pflanzen. extracten hergestellt. Ueber den günstigen Erfolg einer solchen Petroleumseife bei Bekämpfung der Blattläuse auf Rübensamen berichtete in der letzten Zeit Kuntze. ${ }^{1}$ )

Ich empfehle nun zur Herstellung solcher Mittel gegen Pflanzenschädlinge (Aphiden, Cochylis etc.) statt des Petroleums die Verwendung von rohen Petrolsäuren respective deren Salzen und Aethern.

Die rohen Petrolsäuren werden aus der von der

1) Zeitschr. Rübenzuckerind. I898, 753; Chem. Ztg. Rep. 1898, 264. 